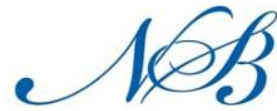

Notulae Scientia Biologicae

Original Article

\title{
Morphology of Some Species in the Subfamily Papilionoideae
}

\author{
Joan Adeola OWOLABI, Olubukola ADEDEJI*
}

Obafemi Awolowo University, Department of Botany, Ile-Ife, Osun State, Nigeria; oadedeji@oauife.edu.ng(*orresponding author)

\begin{abstract}
Morphological study of ten species in the subfamily Papilionoideae was carried out with the view to documenting diagnostic characters that would distinguish or group the species. The species studied belong to four tribes, namely: tribe Desmodieae - Desmodium tortuosum (Sw.) DC., Desmodium scorpiurus (Sw.) Desv., Desmodium adscendens (Sw.) DC., tribe Phaseoleae - Cajanus cajan (L.) Millsp., Calopogonium mucunoides Desv., Centrosema molle (Mart.) ex. Benth., Mucuna pruriens (Linn.) Walp., Vigna unguiculata (Linn.) Walp., tribe Crotalarieae - Crotalaria retusa Linn., tribe Robinieae - Gliricidia sepium (Jacq.) Walp. Qualitative and quantitative traits which had not been documented in previous works, especially in Nigeria, were studied. These include plant life span; leaf/leaflet apex, base, margin and pubescence; stem type, colour, shape and pubescence; sepal colour and pubescence; nature of margin of petal standard and presence or absence of pedicel; fruit colour, pubescence, tip and shape; seed colour, shape, surface and presence or absence of prominent hilum on the seed; number of seeds per fruit; pedicel length; length and width of petal standard, keel and wing. Characters of taxonomic value documented in this study were leaf type, leaf shape, leaf base, petiole type, stem type, seed shape, petal standard length, petal keel length and petal wing width. Data were subjected to one - way analysis of variance using Duncan's multiple range test. It was noted that the important characters that can be used in establishing taxonomic relationship in the sub-family Papilionoideae were leaf type, leaf shape, leaf base, petiole type, stem shape, petal colour, petal margin and seed shape.
\end{abstract}

Keywords: morphology, Papilionoideae, tribe, character, taxonomy

\section{Introduction}

The subfamily Papilionoideae belongs to the family Leguminosae. The subfamily is sometimes recognized as a separate family Papilionaceae. The species of this subfamily are generally identifiable by their characteristic papilionaceous (butterfly-like) flowers (ILDIS, 2005; Cullen et al., 2011). Papilionoideae is the largest group of legumes, with about twothirds of all the genera and species of the family Leguminosae. The homogenous subfamily is also the most widespread, generally distributed throughout the world; it is most numerous and extending further into warmer temperate regions compared with the other two subfamilies, Caesalpinoideae and Mimosoideae, in Leguminosae (Hutchinson and Dalziel, 1958; Gurcharan, 2004; Duane and Paul, 2012). The subfamily consists of about 475 genera and nearly 14,000 species, grouped in 14 tribes (APG, 2012; Duane and Paul, 2012) out of which about 335 species were recorded in Nigeria (Hutchinson and Dalziel, 1954). The members of subfamily Papilionoideae are predominantly herbs or herbaceous climbers; sometimes they are erect or climbing shrubs, trees or lianas (Datta and Mukherii, 1952; Hutchinson and Dalziel, 1958; Watson and Dallwitz, 1999; Gurcharan, 2004; ILDIS, 2005). It is an extremely important subfamily and finds a wide range of usefulness (Datta and Mukherji, 1952; ILDIS, 2005).
Most plants are classified based on their external morphological structures. The morphology and ontogenies of taxa are important for intra-generic systematics (Sayantan and Amal, 2004). Alexander (2004) presented an illustrated survey of abaxial leaf surface of 22 Desmodium species found in North Carolina and a diagnostic key to facilitate the identification and teaching of the species. He reported that Desmodium Desv. (Papilionoideae) is perhaps the most difficult genus among Carolina legumes. El-Gazzar et al. (2013) recorded 81 morphological characters for 226 species and intra-specific taxa belonging to 75 genera representing 21 of the 32 tribes currently recognized in the subfamily Papilionoideae, to know the extent to which the currently accepted classification of the subfamily by Polhill and Raven (1981) would withstand the test of numerical analyses. The authors concluded that the currently accepted circumscription and inter-relationships among the disrupted tribes and genera of Papilionoideae are in need of much more detailed investigation.

A search in the literature also shows that the information about the comparative morphology of most species in the subfamily Papilionoideae, especially those found in Nigeria, is scanty. This study therefore aimed to document the morphological traits of taxonomic value in the species studied. 


\section{Materials and Methods}

For the purpose of this work, ten species belonging to four tribes in the subfamily Papilionoideae were studied. The species studied were as follows: tribe Desmodieae - Desmodium tortuosum (Sw.) DC., Desmodium scorpiurus (Sw.) Desv., Desmodium adscendens (Sw.) DC., tribe Phaseoleae - Cajanus cajan (L.) Millsp., Calopogonium mucunoides Desv., Centrosema molle (Mart.) ex. Benth., Mucuna pruriens (Linn.) Walp., Vigna unguiculata (Linn.) Walp., tribe Crotalarieae - Crotalaria retusa Linn., tribe Robinieae - Gliricidia sepium (Jacq.) Walp.

All plant species were collected from different locations in Ile-Ife, latitude $07^{\circ} 30^{\prime} \mathrm{N}$ and longitude $04^{\circ} 40^{\prime} \mathrm{E}$, Osun State, Nigeria.

Qualitative data observed and recorded were plant habit; plant life span; leaf type; leaf/leaflet shape, base, apex, margin, colour, pubescence; stem type, colour, shape, pubescence; presence or absence of stipule; inflorescence type; sepal aestivation, colour; standard petal margin; fruit type, segmentation, indentation or ribbing; fresh fruit colour; dry fruit colour; fruit shape, tip shape; seed shape and colour; presence or absence of depression on the seed; nature of seed surface; hilum prominent or not prominent.

Quantitative data taken were leaf length and width; petiole length; fruit length and width; number of seeds per fruit; pedicel length; petal standard length and width; petal keel length and width; petal width length and width.

Quantitative data generated from this work were subjected to one - way analysis of variance using Duncan multiple range test to show significant differences. Simple descriptive statistics from SPSS analysis were also used to calculate the minimum, maximum, means and standard error of means for each trait.

\section{Results}

The qualitative and quantitative traits of the species studied are hereby presented. They are summarised on Tables $1-3$, whereas descriptive terminologies used are according to Metcalfe and Chalk (1979).

Photographs of the diagnostic morphological traits of the studied species are presented on Figs. 1 - 10.

\section{Desmodium tortuosum (Sw.) DC. (Fig. 1)}

Habit: Herb/ under shrub (Fig. 1A).

Lifespan: Annual

Leaf: compound (trifoliate), leaflet: $4.10-18.90 \mathrm{~cm}$ long and $2.20-9.90 \mathrm{~cm}$ wide, ovate in shape, acute at base, acute at apex, entire at margin, green, pubescent, stipulate, petiolate, petiole was between $1.70-6.90 \mathrm{~cm}$ long (Fig. 1B).

Stem: erect, woody, solid, green and reddish brown, cylindrical, ringed and pubescent (Fig. 1C).

Inflorescence: Terminal and axillary panicle (Fig. 1D).

Flower: zygomorphic, pedicellate; Sepal: green, polysepalous, ovate in shape, 5 in number, pubescent; Petals: standard: 0.40 $0.50 \mathrm{~cm}$ long and $0.40-0.45 \mathrm{~cm}$ wide, keel: $0.40-0.50 \mathrm{~cm}$ long and $0.50-0.30 \mathrm{~cm}$ wide, wing: $0.40-0.45 \mathrm{~cm}$ long and $0.20-$ $0.25 \mathrm{~cm}$ wide, pinkish purple, papilionaceous shape, 5 in number, pedicel $1.20-1.50 \mathrm{~cm}$ long (Fig. 1E).

Fruit: lomentum, segmented, segment orbicular, deeply indented on both sides twisted, obtuse at apex, $2.30-3.00 \mathrm{~cm}$ long and $0.30-0.40 \mathrm{~cm}$ wide, green with reddish brown pattern when fresh, brown when dry, pubescent, one row of seed per fruit (Fig. 1G and Fig. 1I).
Seed: bean shape, occasionally triangular, light brown to dark brown, shinny, glabrous, depressed around the middle, $5-7$ seeds per fruit (Fig. 1F).

\section{Desmodium scorpiurus (Sw.) Desv. (Fig. 2)}

Habit: herbaceous creeper (Fig. 2A).

Lifespan: perennial.

Leaf: compound (trifoliate), leaflet; $2.20-10.50 \mathrm{~cm}$ long and $1.00-3.90 \mathrm{~cm}$ wide, ovate to elliptic in shape, obtuse at base, acute at apex, entire at margin, green, pubescent, stipulate, petiolate, petiole $2.90-6.90 \mathrm{~cm}$ long (Fig. 2B).

Stem: weak, creeper, herbaceous, roots at nodes, solid, green, slightly twisted, triangular and pubescent (Fig. 2C and D).

Inflorescence: terminal and axillary raceme (Fig. 2E - G).

Flower: zygomorphic, sub sessile; sepal: green, polysepalous, ovate in shape, 5 in number, pubescent; petals: standard of 0.40 $0.50 \mathrm{~cm}$ long and $0.40-0.50 \mathrm{~cm}$ wide, keel: $0.40-0.45 \mathrm{~cm}$ long and $0.20-0.25 \mathrm{~cm}$ wide, wing: $0.40-0.50 \mathrm{~cm}$ long and $0.20-$ $0.25 \mathrm{~cm}$ wide, pinkish purple, papilionaceous, 5 in number, pedicel $0.20-0.30 \mathrm{~cm}$ long (Fig. $2 \mathrm{H}$ ).

Fruit: lomentum, segmented, slightly indented on both sides, cylindrical, obtuse at apex, $2.10-4.30 \mathrm{~cm}$ long and $0.20 \mathrm{~cm}$ wide, light green when fresh, brown when dry, pubescent, one row of seed per fruit (Fig. 2I and K).

Seed: cylindrical, green when fresh, brown when dry, smooth, 4-8 seeds per fruit (Fig. 2J).

\section{Desmodium adscendens (Sw.) DC. (Fig. 3)}

Habit: herbaceous creeper (Fig. 3A).

Life span: perennial.

Leaf: compound (trifoliate), leaflet; $1.80-4.60 \mathrm{~cm}$ long and $1.20-3.30 \mathrm{~cm}$ wide, obovate in shape, obtuse to round at base, round/retuse at apex, entire at margin, green, pubescent, stipulate, petiolate, petiole $1.40-2.50 \mathrm{~cm}$ long (Fig. 3B).

Stem: weak, creeper, herbaceous, roots at nodes, solid, green, cylindrical and pubescent (Fig. 3C and D).

Inflorescence: terminal and axillary raceme (Fig. 3E).

Flower: zygomorphic, pedicellate; sepal: green, polysepalous, ovate in shape, 5 in number, pubescent; petals: standard of 0.40 $0.50 \mathrm{~cm}$ long and $0.50-0.55 \mathrm{~cm}$ wide, keel: $0.40-0.50 \mathrm{~cm}$ long and $0.20-0.25 \mathrm{~cm}$ wide, wing: $0.40-0.50 \mathrm{~cm}$ long and $0.20-$ $0.25 \mathrm{~cm}$ wide, faded pinkish purple, papilionaceous, 5 in number, pedicel $0.6-1.2 \mathrm{~cm}$ long (Fig. 3F and $\mathrm{G}$ ).

Fruit: lomentum, segmented, deeply indented on one side, short aristate at apex, $0.80-2.40 \mathrm{~cm}$ long and $0.25-0.35 \mathrm{~cm}$ wide, deep green when fresh and brown when dry, flat, pubescent, one row of seed per fruit (Fig. $3 \mathrm{H}$ and J).

Seed: bean shape occasionally triangular, green when fresh, brown when dry, glabrous, $1-4$ seeds per fruit (Fig. 3I).

\section{Mucuna pruriens (Linn.) Walp. (Fig. 4)}

Habit: herbaceous twinner (Fig. 4A).

Lifespan: annual.

Leaf: compound (trifoliate), leaflet; $7.90-18.20 \mathrm{~cm}$ long and $4.70-11.10 \mathrm{~cm}$ wide, wide ovate to wide elliptic in shape, lateral leaflets asymmetric, obtuse at base, obtuse at apex, entire at margin, green, pubescent, stipulate, petiolate, petiole $4.40-14.10$ cm long (Fig. 4B).

Stem: weak, twinner, woody, solid, brown, cylindrical, minutely pubescent (Fig. 4F).

Inflorescence: axillary raceme (Fig. 4G).

Flower: zygomorphic, sub sessile; sepal: green, gamosepalous, 
campanulate in shape, pubescent; petals: standard of $2.00-$ $2.20 \mathrm{~cm}$ long and $1.60-1.80 \mathrm{~cm}$ wide, keel: $2.80-2.90 \mathrm{~cm}$ long and $1.40-1.50 \mathrm{~cm}$ wide, wing: $3.10-3.20 \mathrm{~cm}$ long and $1.60-1.70 \mathrm{~cm}$ wide, deep purple, papilionaceous in shape, 5 in number, pedicel $0.40-0.60 \mathrm{~cm}$ long (Fig. 4E).

Fruit: legume, obtuse at apex, sickle shape $8.40-8.80$ $\mathrm{cm}$ long and $2.10-2.30 \mathrm{~cm}$ wide, rusty brown when fresh, brown when dry, pubescent - densely clothed with rusty stinging hairs, one row of seed per fruit (Fig. 4C and D).

Seed: bean shaped with prominent hilum, light brown to dark brown, glabrous, depressed around the middle, $4-5$ seeds per fruit (Fig. 4H).

\section{Calopogonium mucunoides Desv. (Fig. 5)}

Habit: herbaceous creeper (Fig. 5A).

Life span: perennial.

Leaf: compound (trifoliate), leaflet; $5.20-11.60 \mathrm{~cm}$ long and $4.10-7.40 \mathrm{~cm}$ wide, wide ovate to wide elliptic in shape, lateral leaflets asymmetric, obtuse at base, obtuse at apex, entire at margin, green, pubescent, covered with brown rusty hairs, stipulate, petiolate, petiole $5.60-15.40$ cm long (Fig. 5B).

Stem: weak, creeper, woody, roots at nodes, solid, green, cylindrical and pubescent (Fig. 5C and D).

Inflorescence: axillary cluster (Fig. 5E).

Flower: zygomorphic, sessile; sepal: green, polysepalous, ovate in shape, 5 in number, pubescent; petals: standard of $0.50-0.60 \mathrm{~cm}$ long and $0.50-0.60 \mathrm{~cm}$ wide, keel: $0.70-0.80 \mathrm{~cm}$ long and $0.30-0.35 \mathrm{~cm}$ wide, wing: $0.50-0.55 \mathrm{~cm}$ long and $0.20-0.25 \mathrm{~cm}$ wide, bluish lilac, papilionaceous in shape, 5 in number (Fig. 5I).

Fruit: legume, mucronate at apex, $2.90-4.30 \mathrm{~cm}$ long and $0.30-0.50 \mathrm{~cm}$ wide, green when fresh, brown when dry, pubescent, one row of seed per fruit (Fig. 5F and G).

Seed: bean shaped, largely square, light brown to brown, shinny, glabrous, depressed around the middle, 6 8 seeds per fruit (Fig. $5 \mathrm{H}$ ).

\section{Cajanus cajan (L.) Millsp. (Fig. 6)}

Habit: shrub (Fig. 6A).

Lifespan: annual.

Leaf: compound (trifoliate), leaflet; $6.50-13.70 \mathrm{~cm}$ long and $2.30-4.10 \mathrm{~cm}$ wide, elliptic in shape, acute at base, acuminate at apex, entire at margin, green, pubescent, stipulate, petiolate, petiole $3.80-7.00 \mathrm{~cm}$ long (Fig. 6B).

Stem: erect, woody, solid, green, angular-cylindrical (angles looking like white straight lines), pubescent (Fig. $6 \mathrm{E})$.

Inflorescence: axillary cluster (Fig. 6D).

Flower: zygomorphic, pedicellate; sepal: green, gamosepalous, campanulate in shape, pubescent; Petals: standard of $1.60-1.70 \mathrm{~cm}$ long and $1.60-1.70 \mathrm{~cm}$ wide, keel: $1.50-1.60 \mathrm{~cm}$ long and $0.70-0.80 \mathrm{~cm}$ wide, wing: $1.30-1.40 \mathrm{~cm}$ long and $0.60-0.70 \mathrm{~cm}$ wide, bright yellow with reddish brown veining at the back of the standard, indented at margin of the standard, papilionaceous in shape, 5 in number, pedicel $0.90-1.40$ cm long (Fig. 6F).

Fruit: legume, ribbed, acuminate at apex, $5.70-6.90$ $\mathrm{cm}$ long and $0.70-0.90 \mathrm{~cm}$ wide, green with reddish brown pattern when fresh, brown when dry, pubescent, one row of seed per fruit (Fig. 6G).
Seed: orbicular with prominent hilum, greyishbrown, rough, shinny, glabrous, 3 - 6 seeds per fruit (Fig. 6C).

\section{Crotalaria retusa Linn. (Fig. 7)}

Habit: shrub (Fig. 7A).

Lifespan: annual.

Leaf: simple, leaf of $4.70-10.10 \mathrm{~cm}$ long and $2.10-3.60 \mathrm{~cm}$ wide, oblanceolate in shape, cuneate at base, retuse at apex, entire at margin, green, minutely pubescent, stipulate and sub sessile, petiole $0.20-0.30 \mathrm{~cm}$ long (Fig. $7 \mathrm{~B}$ ).

Stem: erect, woody, hollow, green, cylindrical with vertical ridges, minutely pubescent (Fig. $7 \mathrm{C}$ ).

Inflorescence: terminal raceme (Fig. 7D).

Flower: zygomorphic, sub sessile; sepal: green, gamosepalous, campanulate in shape, minutely pubescent; petals: standard: 2.20 $-2.40 \mathrm{~cm}$ long and $2.70-2.90 \mathrm{~cm}$ wide, keel: $1.40-1.60 \mathrm{~cm}$ long and $1.20-1.30 \mathrm{~cm}$ wide, wing: $1.40-1.60 \mathrm{~cm}$ long and $1.00-1.20 \mathrm{~cm}$ wide, bright yellow with reddish-brown veining on the standard alone, papilionaceous in shape, 5 in number, pedicel $0.80-1.00 \mathrm{~cm}$ long(Fig. 7E).

Fruit: legume, aristate curved at apex, oblong-cylindrical 3.30 $-4.40 \mathrm{~cm}$ long and $0.90-1.30 \mathrm{~cm}$ wide, green when fresh, black when dry, glabrous, one row of seed per fruit (Fig. $7 F$ and $G$ ).

Seed: reniform in shape, dark brown, glabrous, $9-22$ seeds per fruit (Fig. 7H).

\section{Vigna unguiculata (Linn.) Walp. (Fig. 8)}

Habit: herbaceous creeper or climber (Fig. 8A).

Lifespan: perennial.

Leaf: compound (trifoliate), leaflet; $5.30-14.70 \mathrm{~cm}$ long and $3.20-7.80 \mathrm{~cm}$ wide, ovate, lateral leaflets asymmetric, acute at base, sharp acute at apex, entire at margin, green, minutely pubescent, stipulate, petiolate, petiole $6.00-17.00 \mathrm{~cm}$ long (Fig. 8B).

Stem: weak, creeper or climber, roots at nodes, herbaceous, solid, green with reddish-brown patch, cylindrical and glabrous (Fig. 8C).

Inflorescence: axillary cluster (Fig. $8 \mathrm{H}$ ).

Flower: zygomorphic, sessile; sepal: green, gamosepalous, campanulate, glabrous; petals: standard: $2.50-2.70 \mathrm{~cm}$ long and $3.30-3.50 \mathrm{~cm}$ wide, keel: $2.40-2.60 \mathrm{~cm}$ long and $1.50-1.70$ $\mathrm{cm}$ wide, wing: $2.00-2.20 \mathrm{~cm}$ long and $1.40-1.60 \mathrm{~cm}$ wide, lilac, papilionaceous in shape, 5 in number (Fig. 8E).

Fruit: legume, obtuse at apex, cylindrical, $7.30-11.60 \mathrm{~cm}$ long and $0.30-0.40 \mathrm{~cm}$ wide, green when fresh, black when dry, glabrous, one row of seed per fruit (Fig. 8D and F).

Seed: bean-shaped, dark-brown with black patches, glabrous, $6-17$ seeds per fruit (Fig. 8G)

\section{Centrosema molle Mart. ex. Benth. (Fig. 9)}

Habit: herbaceous climber or creeper (Fig. 9A and B).

Lifespan: perennial.

Leaf: compound (trifoliate), leaflet; $5.00-7.40 \mathrm{~cm}$ long and $3.00-4.60 \mathrm{~cm}$ wide, ovate in shape, obtuse at base, acuminate at apex, entire at margin, dark green, minutely pubescent, stipulate, petiolate, petiole $2.00-6.00 \mathrm{~cm}$ long (Fig. 9C and Fig. 9D).

Stem: weak, creeper or climber, woody, root at nodes, solid, brown, twisted, minutely pubescent (Fig. 9G).

Inflorescence: axillary cluster.

Flower: zygomorphic, sub sessile; sepal: green, gamosepalous, campanulate in shape, minutely pubescent; 
240

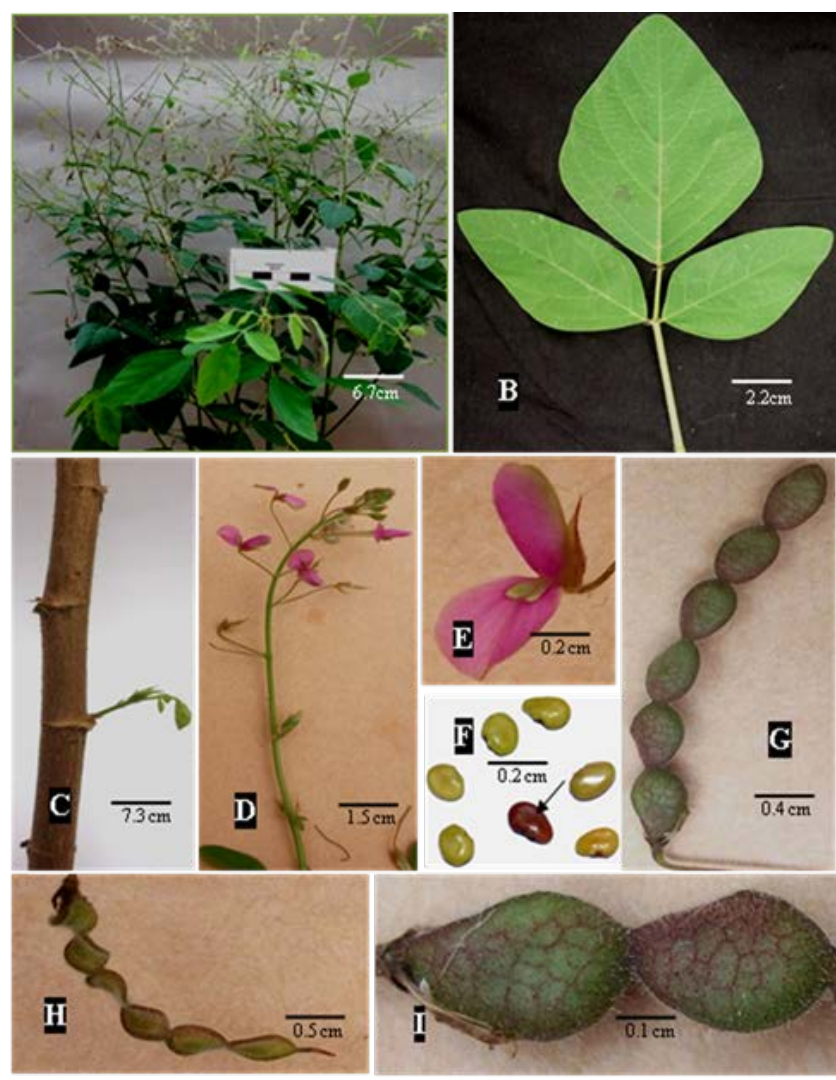

Fig. 1.Morphology of Desmodium tortuosum (Sw.) DC

A. Habit(herb/under shrub), B. Leaf, C.Stem,D. Inflorescence, E. Flower,F. Seeds (arrow points at depression), G. Fruit (fresh), H. Fruit (dry and twisted), I. Fruit (showing reddish brown pattern and hairs on fruits

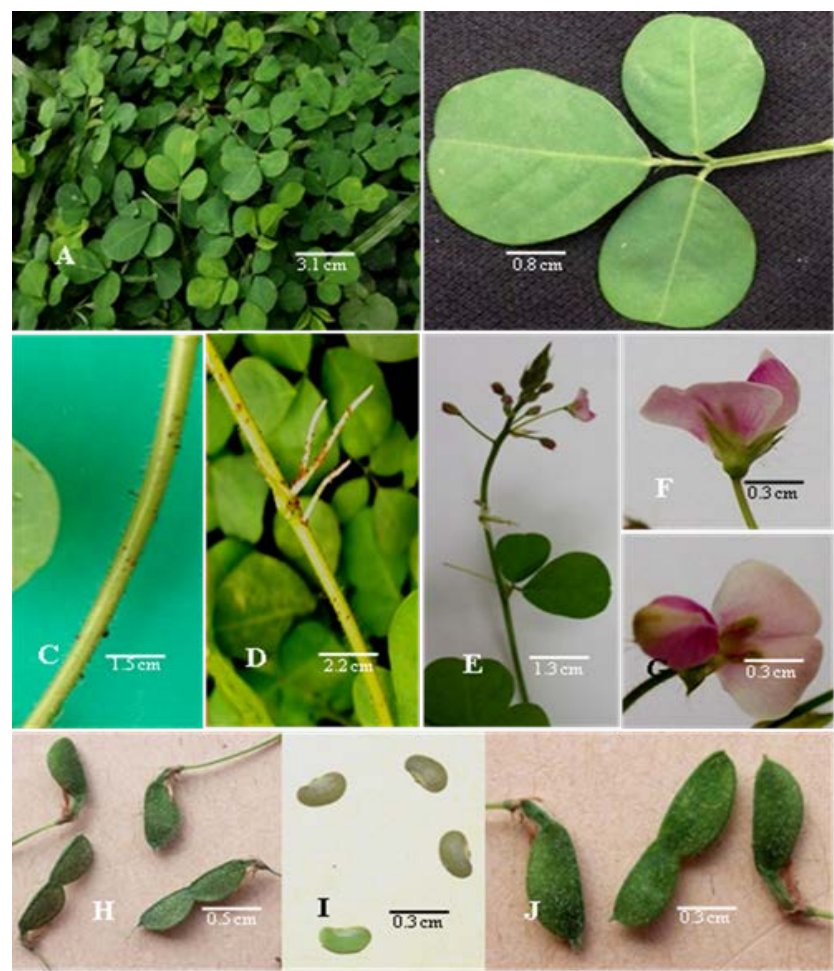

Fig. 3. Morphology of Desmodium adscendens (Sw.) DC.

A. Habit (herbaceous creeper), B. Leaf, C. Stem, D. Stem rooting at node, E. Inflorescence, F - G. Flowers, H. Fruits, I. Seeds (fresh and dry), J.Fruits (showing hairs)

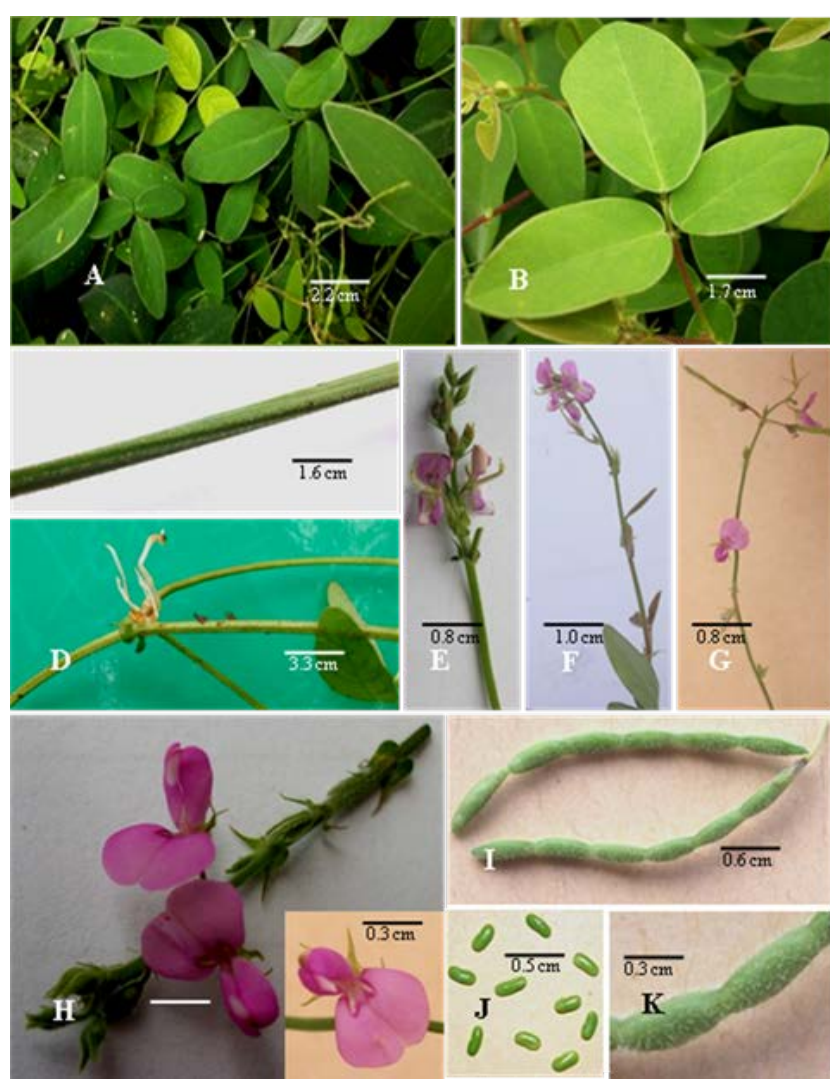

Fig. 2. Morphology of Desmodium scorpiurus (Sw.) Desv

A. Habit (herbaceous creeper), B. Leaf, C. Stem, D. Stem (rooting at node), E - G. Inflorescences, H. Flowers, I. Fruits (fresh), J. Seeds, K. Fruits (showing hairs)

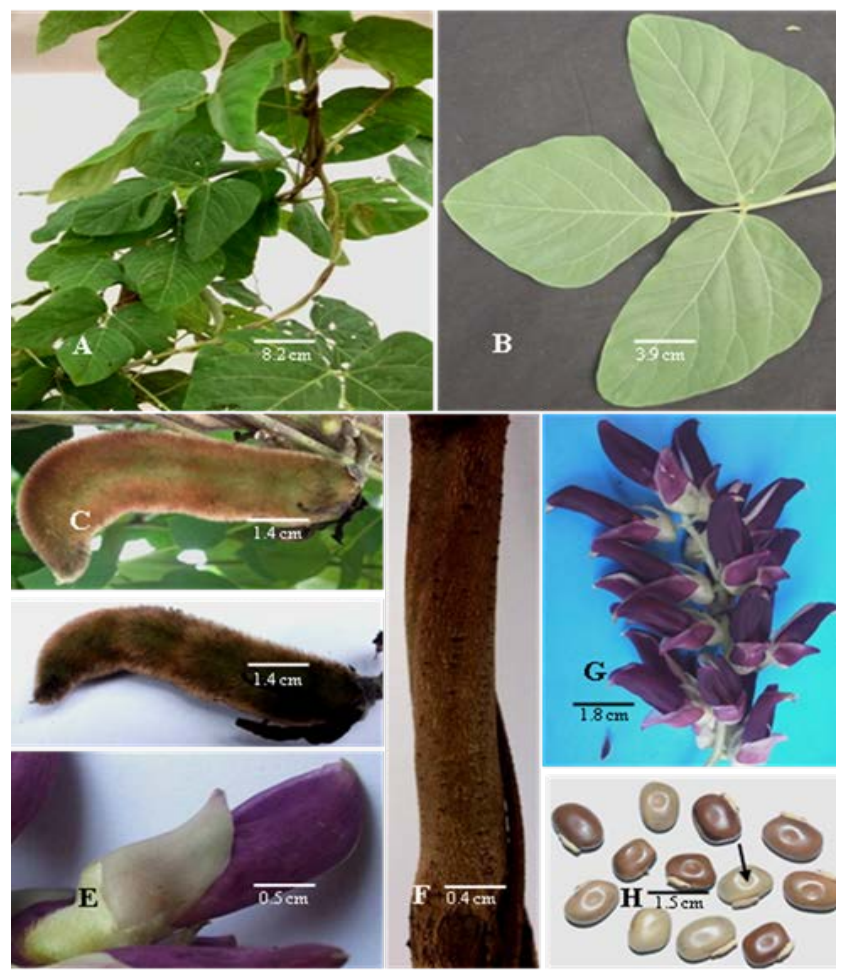

Fig. 4. Morphology of Mucuna pruriens (Linn.) Walp.

A. Habit (herbaceous twinner), B. Leaf, C. Fruits, D. Fruits, E. Flower, F. Stem, G. Inflorescence, H. Seeds (arrow points at depression) 


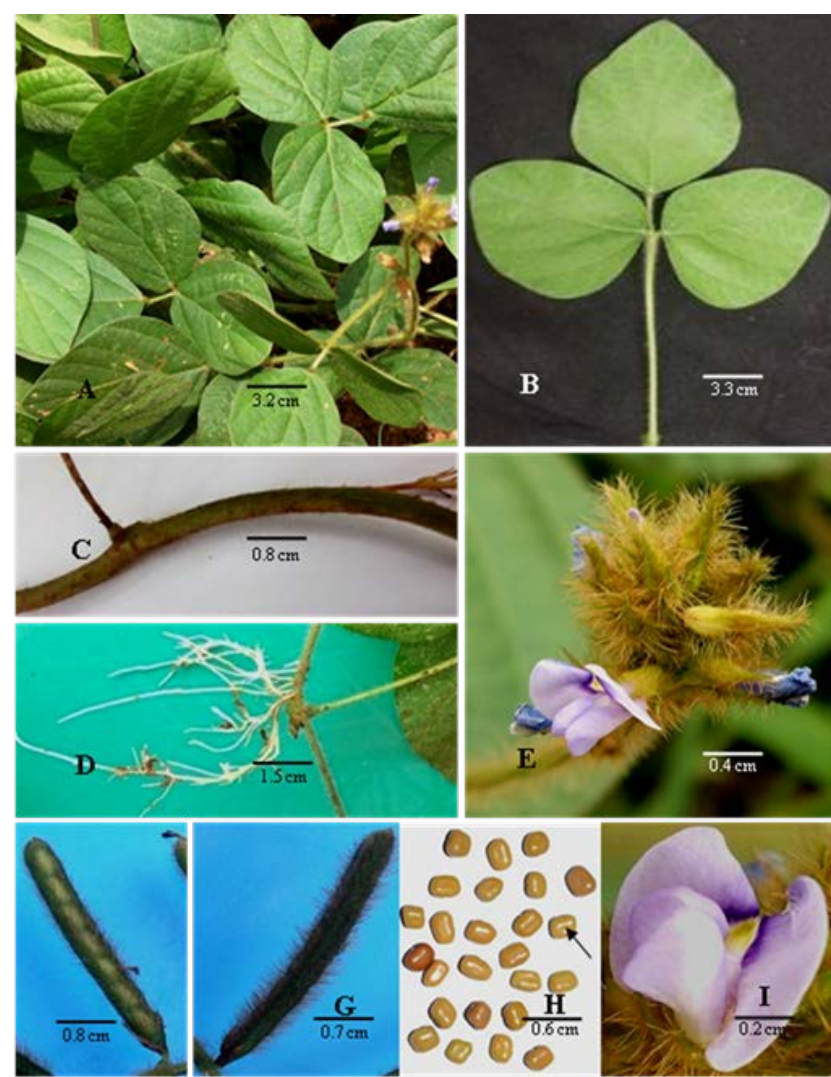

Fig. 5. Morphology of Calopogonium mucunoides Desv.

A. Habit (herbaceous creeper), B. Leaf, C. Stem, D. Stem (rooting at node), E. Inflorescence (axillary cluster), F. Fruit (fresh), G. Fruit (showing hairs), H. Seeds (arrow points at depression), I. Flower
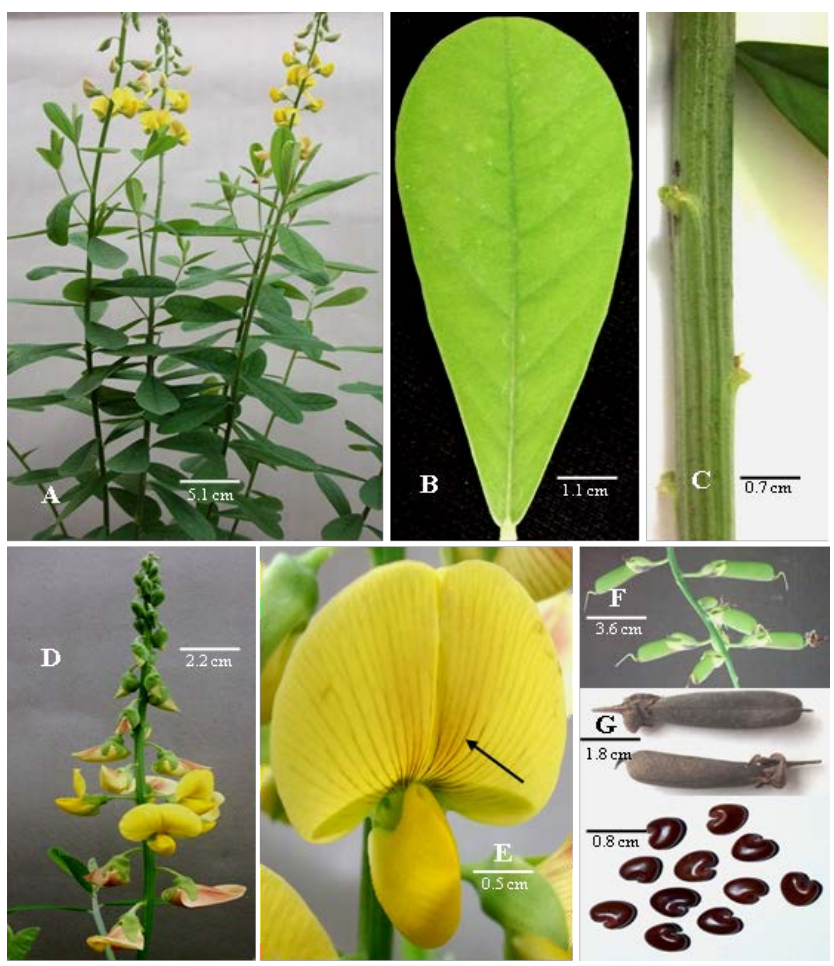

Fig. 7. Morphology of Crotalaria retusa:Linn.

A. Habit (shrub), B. Leaf, C. Stem, D. Inflorescence (terminal raceme), E. Flower (arrow points at reddish-brown veining on the standard alone), F. Fruit (fresh), G. Fruit (dry), H. Seeds

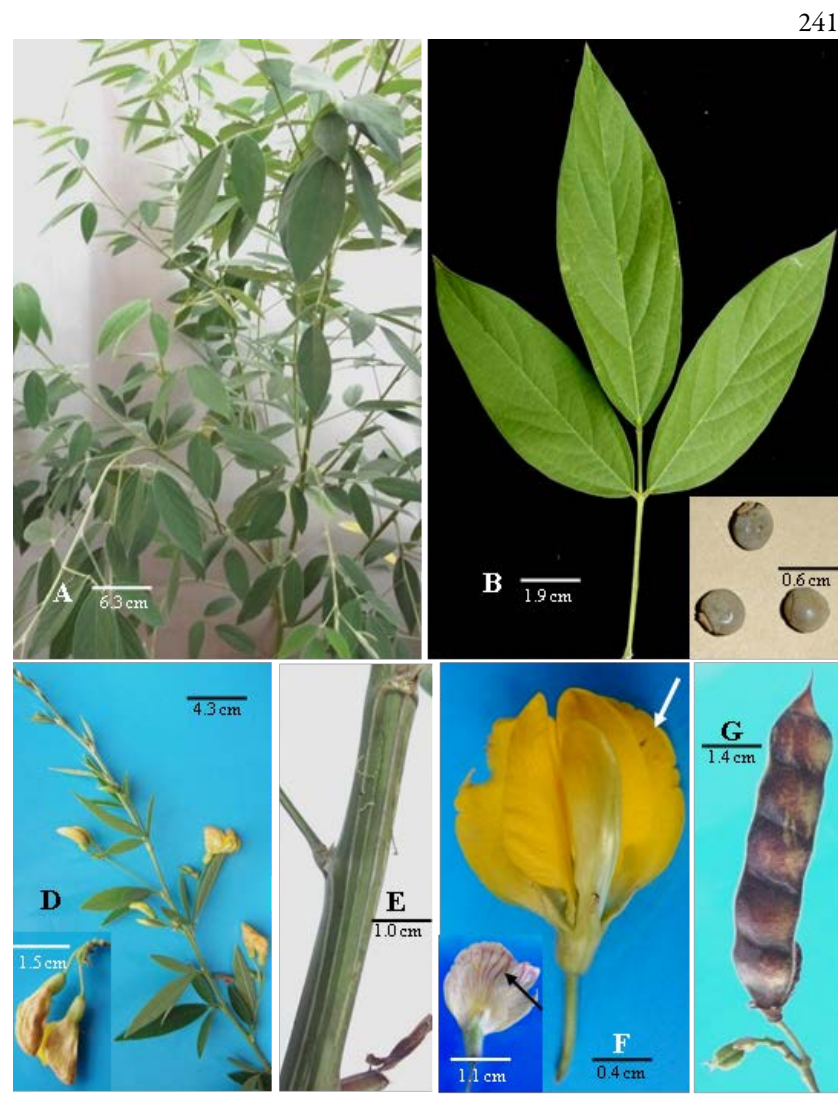

Fig. 6. Morphology of Cajanus cajan (L.) Millsp.

A. Habit (shrub), B. Leaf, C. Seeds, D. Inflorescence (axillary cluster), E. Stem, F. Flower (black arrow points at reddish-brown veining and red arrow points at indented margin of the standard), G. Fruit (fresh)

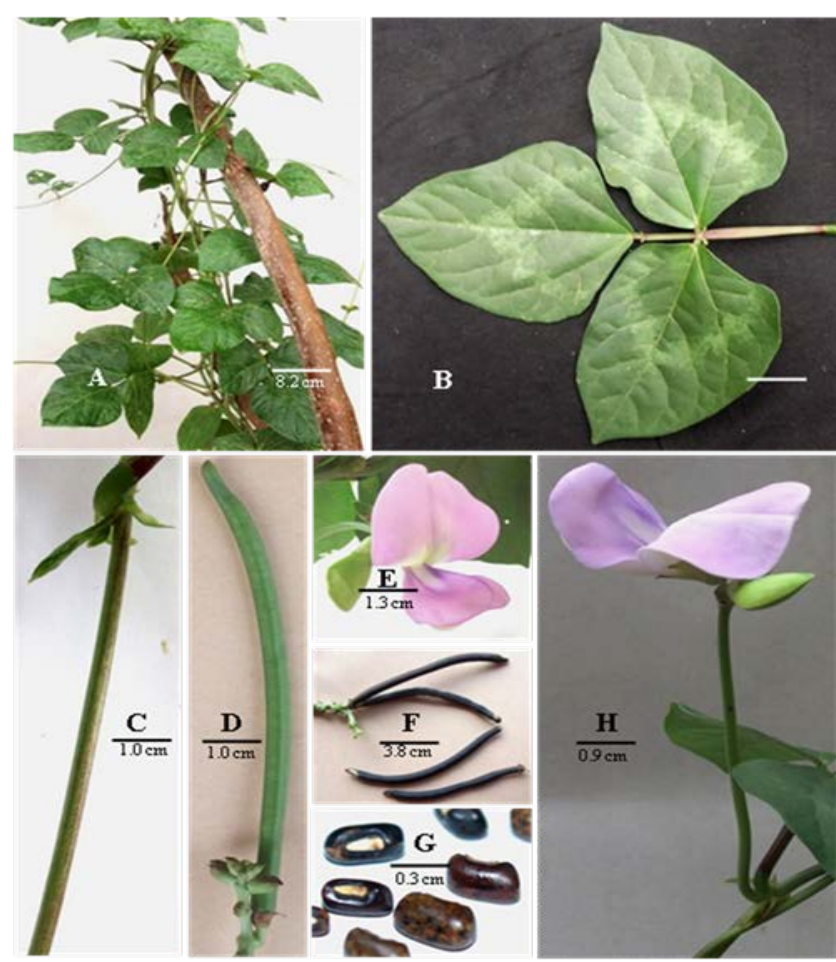

Fig. 8. Morphology of Vigna unguiculata (Linn.) Walp.

A. Habit (herbaceous creeper or climber), B. Leaf, C. Stem, D. Fruit (fresh), E. Flower, F. Fruits (dry), G. Seeds, H. Inflorescence (axillary cluster) 


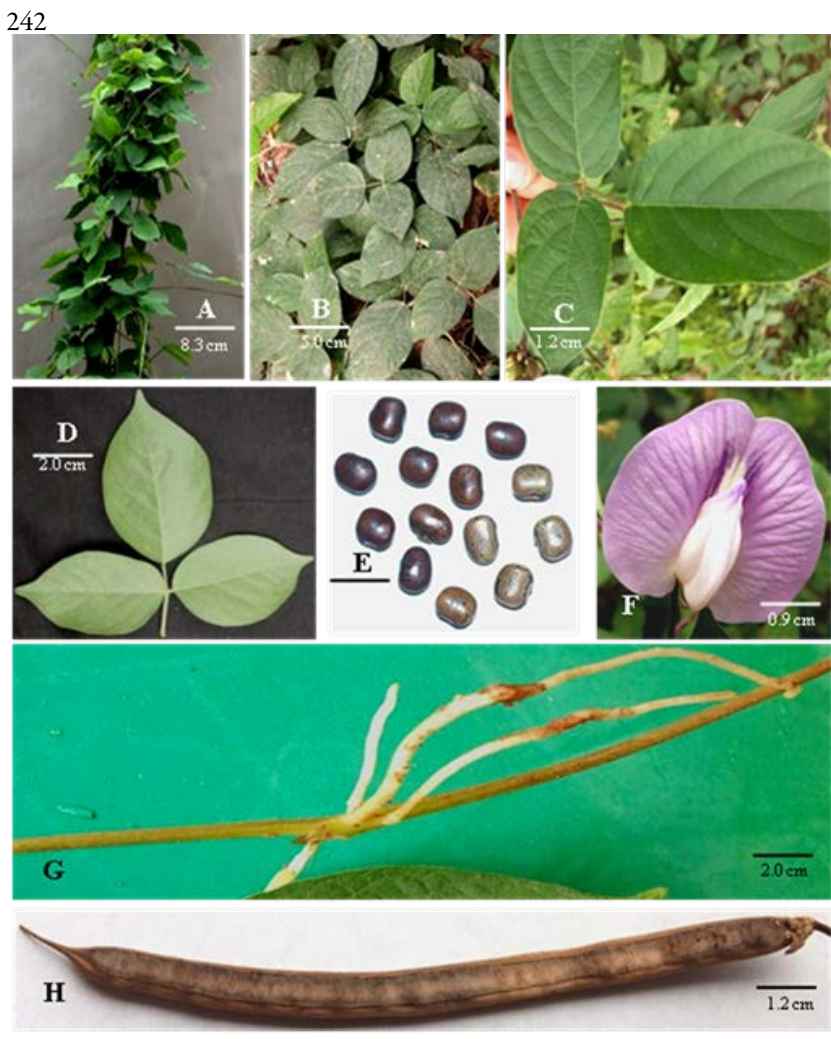

Fig. 9. Morphology of Centrosema molle Mart. ex. Benth.

$\mathrm{A}$ and B. Habit (herbaceous creeper or climber, A as a climber, B as a creeper), C and D. Leaves, E. Seeds, F. Flower, G. Stem (rooting at node), H. Fruit (dry)

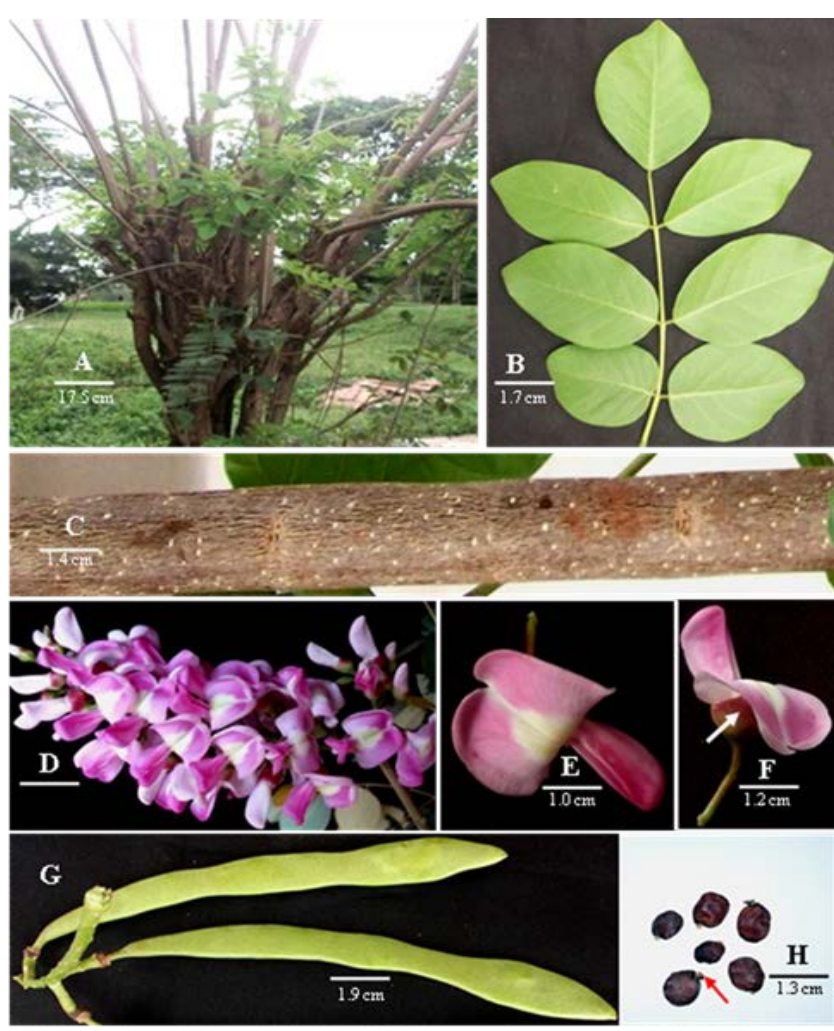

Fig. 10. Morphology of Gliricidia sepium (Jacq.) Walp.

A. Habit (tree), B. Leaf, C. Stem, D. Inflorescence (terminal panicle), E and F. Flower (arrow pointing at the calyx), G. Fruit (fresh), H. Seeds (arrow pointing at prominent hilum

Table 1. Summary of the qualitative morphological traits of the studied species of the subfamily Papilionoideae

\begin{tabular}{|c|c|c|c|c|c|c|c|c|}
\hline Species & $\begin{array}{l}\text { Plant } \\
\text { habit }\end{array}$ & Lifespan & Leaf type & Leaf/Leaflet shape & Lateral leaflet & Leafapex & Leafbase & Leaf margin \\
\hline Desmodium tortuosum & Shrub & Annual & Trifoliate & Ovate & Symmetric & Obtuse & Obtuse & Entire \\
\hline Desmodium scorpiurus & Herb & Perennial & Trifoliate & Elliptic & Symmetric & Obtuse & Obtuse & Entire \\
\hline Desmodium adscendens & Herb & Perennial & Trifoliate & Obovate & Symmetric & Retuse & Obtuse & Entire \\
\hline Mucuna pruriens & Herb & Perennial & Trifoliate & Wide ovate/wide elliptic & Asymmetric & Obtuse & Obtuse & Entire \\
\hline Calopogonium mucunoides & Herb & Perennial & Trifoliate & Wide ovate/wide elliptic & Asymmetric & Obtuse & Obtuse & Entire \\
\hline Cajanus cajan & Shrub & Annual & Trifoliate & Elliptic & Symmetric & Acuminate & Acute & Entire \\
\hline Crotalariaretusa & Shrub & Annual & Simple & Oblanceolate & Symmetric & Retuse & Cuneate & Entire \\
\hline Vigna unguiculata & Herb & Perennial & Trifoliate & Ovate & Asymmetric & Acute & Acute & Entire \\
\hline Centrosemamolle & Herb & Perennial & Trifoliate & Ovate & Symmetric & Acuminate & Obtuse & Entire \\
\hline Gliricidia sepium & Tree & Perennial & Unipinnate & Elliptic & Symmetric & Acute & Obtuse & Entire \\
\hline
\end{tabular}

Table 1. Summary of the qualitative morphological traits of the studied species of the subfamily Papilionoideae (Contd)

\begin{tabular}{|c|c|c|c|c|c|c|c|}
\hline Species & $\begin{array}{c}\text { Leaf } \\
\text { colour }\end{array}$ & Leaf pubescence & Stipule & Petiole & Stem & Stem type & Stem colour \\
\hline Desmodium tortuosum & Green & Minutely pubescent & + & + & Erect & Woody/Solid & Green/reddish brown \\
\hline Desmodium scorpiurus & Green & Pubescent & + & + & Weak / root at node & Herbaceous/Solid & Green \\
\hline Desmodium adscendens & Green & Pubescent & + & + & Weak / root at node & Herbaceous/Solid & Green \\
\hline Mucuna pruriens & Green & Pubescent & + & + & Weak & Woody/Solid & Brown \\
\hline Calopogonium mucunoides & Green & Pubescent & + & + & Weak/ root at node & Woody/Solid & Green \\
\hline Cajanus cajan & Green & Pubescent & + & + & Erect & Woody/Solid & Green \\
\hline Crotalaria retusa & Green & Minutely pubescent & + & \pm & Erect & Woody/Hollow & Green \\
\hline Vigna unguiculata & Green & Minutely pubescent & + & + & Weak/ root at node & Herbaceous/Solid & Green/ reddish brown \\
\hline Centrosema molle & Green & Minutely pubescent & + & + & Weak/ root at node & Woody/Solid & Brown \\
\hline Gliricidia sepium & Green & Glabrous & - & + & Erect & Woody/Solid & Brown/white spot \\
\hline
\end{tabular}

Key: + : Present; - : Absent; \pm : Sub sessile 
Table 1. Summary of the qualitative morphological traits of the studied species of the subfamily Papilionoideae (Contd)

\begin{tabular}{lcccccccc}
\hline Species & Petal colour & $\begin{array}{c}\text { Standard } \\
\text { margin }\end{array}$ & Flower & Pedicel & Fruit & Fruit segment & $\begin{array}{c}\text { Fresh Fruit } \\
\text { colour }\end{array}$ & $\begin{array}{c}\text { Dry Fruit } \\
\text { Colour }\end{array}$ \\
\hline Desmodium tortuosum & Pinkishpurple & Entire & Zygomorphic & Pedicellate & Lomentum & Bothsidesindented & Green/Red & Brown \\
Desmodium scorpiurus & Pinkishpurple & Entire & Zygomorphic & Subsessile & Lomentum & Bothsidesindented & Green & Brown \\
Desmodium adscendens & Fadedpinkishpurple & Entire & Zygomorphic & Pedicellate & Lomentum & Onesideindented & Green & brown \\
Mucunapruniens & Deeppurple & Entire & Zygomorphic & Subsessile & Legume & Ribbed & Brown/green & Brown \\
Calopogonium mucunoides & Bluishlilac & Entire & Zygomorphic & Sessile & Legume & Notribbed & Green & Brown \\
Cajanus cajan & Yellow/redveining & Indented & Zygomorphic & Pedicellate & Legume & Ribbed & Green/Red & Brown \\
Crotalaria retusa & Yellow/redveining & Entire & Zygomorphic & Subsessile & Legume & Notribbed & Green & Black \\
Vigna unguiculata & Lilac & Entire & Zygomorphic & Sessile & Legume & Notribbed & Green & Black \\
Centrosemamolle & Lilac/violetveining & Entire & Zygomorphic & Subsessile & Legume & Notribbed & Green & Brown \\
Glinicidiasepium & Pinkishpurple/white & Entire & Zygomorphic & Subsessile & Legume & Notribbed & Green & Brown \\
\hline
\end{tabular}

Table 1. Summary of qualitative morphological characters of the species of the subfamily Papilionoideae studied (Contd)

\begin{tabular}{|c|c|c|c|c|c|c|c|c|}
\hline Species & $\begin{array}{c}\text { Fruit } \\
\text { Pubescence }\end{array}$ & Fruittip & Fruitshape & Seedshape & SeedColour & $\begin{array}{c}\text { Seed } \\
\text { Depression }\end{array}$ & $\begin{array}{c}\text { Seed } \\
\text { Surface }\end{array}$ & Hilum \\
\hline Desmodium tortuosum & Pubescent & Obtuse & Flat/Twisted & Bean-shaped & Brown & Depressed & Smooth & Notprominent \\
\hline Desmodium scorpiurus & Pubescent & Obtuse & Cylindrical & Cylindrical & Brown & Notdepressed & Smooth & Notprominent \\
\hline Desmodium adscendens & Pubescent & Shortaristate & Flat & Bean-shaped & Brown & Notdepressed & Rough & Notprominent \\
\hline Mucunapruriens & Pubescent & Obtuse & Sickle-shaped & Bean-shaped & Light-darkbrown & Depressed & Smooth & Prominent \\
\hline Calopogonium mucunoides & Pubescent & Mucronate & Flat & Bean-shaped & Light-darkbrown & Depressed & Smooth & Notprominent \\
\hline Cajanus cajan & Pubescent & Acuminate & Notflat & Orbicular & Greyishbrown & Notdepressed & Rough & Prominent \\
\hline Crotalaria retusa & Glabrous & Aristate & Oblong/cylindrical & Heart-shaped & Darkbrown & Notdepressed & Smooth & Notprominent \\
\hline Vigna unguiculata & Glabrous & Obtuse & Cylindrical & Bean-shaped & Darkbrown/blackpatches & Notdepressed & Smooth & Notprominent \\
\hline Centrosema molle & Glabrous & Aristate & Flat & Bean-shaped & Light/Darkbrown+blackpatches & Notdepressed & Smooth & Notprominent \\
\hline Gliricidia sepium & Glabrous & Obtuse & Flat & Orbicular & Darkbrown & Depressed & Rough & Prominent \\
\hline
\end{tabular}

Table 2. Minimum, mean, standard error of mean and maximum values of the quantitative morphological traits of Papilionoideae species studied

\begin{tabular}{|c|c|c|c|c|c|c|}
\hline Species & Leaflength $(\mathrm{cm})$ & Leafwidth $(\mathrm{cm})$ & Petiolelength $(\mathrm{cm})$ & Fruit length $(\mathrm{cm})$ & Fruitwidth $(\mathrm{cm})$ & Noof seeds per fruit \\
\hline & $\operatorname{Min}(\operatorname{Mean} \pm$ SEMM $) \operatorname{Max}$ & $\operatorname{Min}(\operatorname{Mean} \pm$ SEM $)$ Max & $\operatorname{Min}(\operatorname{Mean} \pm$ S.E.M $)$ Max & $\operatorname{Min}(\operatorname{Mean} \pm$ SE.M $) \operatorname{Max}$ & $\operatorname{Min}(\operatorname{Mean} \pm S E M) M a x$ & $\operatorname{Min}(\operatorname{Mean} \pm$ SEMM $) \operatorname{Max}$ \\
\hline Desmodium tortuosum & $4.10(11.06 \pm 1.00) 18.90$ & $220(5.57 \pm 0.45) 9.90$ & $1.70(3.94 \pm 0.27) 6.90$ & $230(256 \pm 0.04) 3.00$ & $0.30(0.32 \pm 0.01) 0.40$ & $5.00(6.00 \pm 0.10) 7.00$ \\
\hline Desmodium scorpiurus & $2.20(6.26 \pm 0.50) 10.50$ & $1.00(2.67 \pm 0.17) 3.90$ & $290(5.15 \pm 0.2) 6.90$ & $2.10(3.30 \pm 0.14) 430$ & $0.20(0.21 \pm 0.00) 0.25$ & $4.00(5.85 \pm 0.32) 8.00$ \\
\hline Desmodium adscendens & $1.80(294 \pm 0.18) 4.60$ & $1.20(2.13 \pm 0.12) 3.30$ & $1.40(1.76 \pm 0.06) 250$ & $0.80(1.35 \pm 0.11) 240$ & $0.25(0.30 \pm 0.00) 0.35$ & $1.00(2.22 \pm 0.22) 4.00$ \\
\hline Mucunapruriens & $7.90(12.90 \pm 0.67) 18.20$ & $4.70(7.93 \pm 0.46) 11.10$ & $4.40(9.52 \pm 0.61) 14.10$ & $8.40(8.58 \pm 0.0)-8.80$ & $210(222 \pm 0.02) 230$ & $400(4.45 \pm 0.11) 5.00$ \\
\hline Calopogoniummuncunoides & $5.20(7.94 \pm 0.47) 11.60$ & $4.10(5.70 \pm 0.22) 7.40$ & $5.60(9.84 \pm 0.53) 15.4$ & $290(3.53 \pm 0.07) 430$ & $0.30(0.43 \pm 0.0) 0.50$ & $6.00(6.75 \pm 0.14) 8.00$ \\
\hline Cajanuscajan & $6.50(10.07 \pm 0.50) 13.70$ & $230(3.09 \pm 0.1) 4.10$ & $3.80(5.57 \pm 0.19) 7.00$ & $5.70(6.29 \pm 0.09) 6.90$ & $0.70(0.79 \pm 0.01) 0.90$ & $3.00(4.55 \pm 0.15) 6.00$ \\
\hline Crotalariaretusa & $4.70(8.15 \pm 0.35) 10.10$ & $210(2.99 \pm 0.10) 3.60$ & $0.20(0.25 \pm 0.01) 0.30$ & $3.30(3.91 \pm 0.06) 440$ & $0.90(1.05 \pm 0.02) 1.30$ & $9.00(14.70 \pm 0.74) 2200$ \\
\hline Vignaungriculata & $5.30(10.01 \pm 0.51) 14.70$ & $3.20(6.16 \pm 0.24) 7.80$ & $6.00(1225 \pm 0.61) 17.00$ & $730(8.92 \pm 0.24) 11.60$ & $0.30(0.39 \pm 0.01) 0.40$ & $6.00(12.10 \pm 0.62) 17.00$ \\
\hline Centrosemamolle & $5.00(6.20 \pm 0.17) 7.40$ & $3.00(3.75 \pm 0.12) 4.60$ & $2.00(3.81 \pm 0.24) 6.00$ & $8.20(13.76 \pm 0.51) 16.60$ & $0.40(0.61 \pm 0.02) 0.80$ & $11.00(13.00 \pm 0.43) 16.00$ \\
\hline Gliricidiasepium & $4.30(7.69 \pm 0.41) 10.60$ & $3.20(4.62 \pm 0.21) 6.30$ & $3.10(3.86 \pm 0.09) 460$ & $1230(15.24 \pm 0.47) 19.50$ & $1.50(1.92 \pm 0.05) 220$ & $4.00(7.10 \pm 0.36) 10.00$ \\
\hline
\end{tabular}

Key: Min=Minimum value, Max=Maximum value, S.E.M=Standard error of mean

Table 3. Species grouping from Duncan's multiple range test based on morphology

\begin{tabular}{|c|c|c|c|c|c|c|c|c|c|c|c|c|c|}
\hline Species & $\begin{array}{l}\text { Leaf } \\
\text { length } \\
(\mathrm{cm})\end{array}$ & $\begin{array}{l}\text { Leaf } \\
\text { width } \\
(\mathrm{cm})\end{array}$ & $\begin{array}{l}\text { Petiole } \\
\text { length } \\
(\mathrm{cm})\end{array}$ & $\begin{array}{l}\text { Fruit } \\
\text { length } \\
(\mathrm{cm})\end{array}$ & $\begin{array}{l}\text { Fruit } \\
\text { width } \\
(\mathrm{cm})\end{array}$ & $\begin{array}{c}\text { No of } \\
\text { seeds per } \\
\text { fruit }\end{array}$ & $\begin{array}{l}\text { Pedicel } \\
\text { length } \\
(\mathrm{cm})\end{array}$ & $\begin{array}{c}\text { Petal } \\
\text { standard } \\
\text { length } \\
(\mathrm{cm}) \\
\end{array}$ & $\begin{array}{c}\text { Petal } \\
\text { standard } \\
\text { width } \\
(\mathrm{cm}) \\
\end{array}$ & $\begin{array}{l}\text { Petal } \\
\text { keel } \\
\text { length } \\
(\mathrm{cm}) \\
\end{array}$ & $\begin{array}{c}\text { Petal } \\
\text { keel } \\
\text { width } \\
(\mathrm{cm}) \\
\end{array}$ & $\begin{array}{l}\text { Petal } \\
\text { wing } \\
\text { length } \\
(\mathrm{cm})\end{array}$ & $\begin{array}{l}\text { Petal } \\
\text { wing } \\
\text { width } \\
(\mathrm{cm}) \\
\end{array}$ \\
\hline $\begin{array}{l}\text { Desmodium } \\
\text { tortuosum }\end{array}$ & $11.06^{\mathrm{b}}$ & $5.57^{\mathrm{b}}$ & $3.94^{\mathrm{d}}$ & $2.56^{\mathrm{f}}$ & $0.32^{\mathrm{g}}$ & $6.00^{\mathrm{cd}}$ & $1.37^{\mathrm{a}}$ & $0.48^{\mathrm{f}}$ & $0.41^{\mathrm{h}}$ & $0.48^{\mathrm{h}}$ & $0.29^{e}$ & $0.41^{\mathrm{g}}$ & $0.21^{\mathrm{g}}$ \\
\hline $\begin{array}{l}\text { Desmodium } \\
\text { scorpiurus }\end{array}$ & $6.26^{\mathrm{d}}$ & $2.67^{\mathrm{ef}}$ & $5.15^{\mathrm{c}}$ & $3.30^{\mathrm{e}}$ & $0.21^{\mathrm{h}}$ & $5.85^{\mathrm{d}}$ & $0.22^{\mathrm{e}}$ & $0.47^{f}$ & $0.47^{\text {gh }}$ & $0.41^{\mathrm{h}}$ & $0.21^{\mathrm{f}}$ & $0.47^{\mathrm{fg}}$ & $0.21^{\mathrm{g}}$ \\
\hline $\begin{array}{l}\text { Desmodium } \\
\text { adscendens }\end{array}$ & $2.94^{\mathrm{e}}$ & $2.13^{\mathrm{f}}$ & $1.76^{\mathrm{e}}$ & $1.35^{\mathrm{g}}$ & $0.30^{g}$ & $2.20^{\mathrm{f}}$ & $0.93^{c}$ & $0.48^{\mathrm{f}}$ & $0.51^{\mathrm{fg}}$ & $0.48^{\mathrm{h}}$ & $0.21^{\mathrm{f}}$ & $0.48^{\mathrm{fg}}$ & $0.21^{\mathrm{g}}$ \\
\hline $\begin{array}{l}\text { Mucuna } \\
\text { pruriens }\end{array}$ & $12.90^{\mathrm{a}}$ & $7.93^{a}$ & $9.52^{\mathrm{b}}$ & $8.58^{c}$ & $2.22^{\mathrm{a}}$ & $4.45^{\mathrm{e}}$ & $0.50^{\mathrm{d}}$ & $2.13^{\mathrm{d}}$ & $1.74^{\mathrm{e}}$ & $2.88^{a}$ & $1.46^{\mathrm{b}}$ & $3.16^{a}$ & $1.65^{\mathrm{a}}$ \\
\hline $\begin{array}{l}\text { Calopogonium } \\
\text { mucunoides }\end{array}$ & $7.94^{\mathrm{c}}$ & $5.70^{\mathrm{b}}$ & $9.84^{\mathrm{b}}$ & $3.53^{\mathrm{e}}$ & $0.43^{\mathrm{f}}$ & $6.75^{\mathrm{cd}}$ & $0.00^{\mathrm{f}}$ & $0.58^{\mathrm{f}}$ & $0.58^{\mathrm{f}}$ & $0.77^{\mathrm{g}}$ & $0.31^{\mathrm{e}}$ & $0.51^{\mathrm{f}}$ & $0.21^{\mathrm{g}}$ \\
\hline Cajanus cajan & $10.07^{b}$ & $3.09^{\mathrm{de}}$ & $5.57^{\mathrm{c}}$ & $6.29^{d}$ & $0.79^{d}$ & $4.55^{\mathrm{e}}$ & $1.08^{\mathrm{b}}$ & $1.69^{\mathrm{e}}$ & $1.68^{\mathrm{e}}$ & $1.59^{\mathrm{e}}$ & $0.78^{\mathrm{d}}$ & $1.38^{\mathrm{e}}$ & $0.68^{\mathrm{e}}$ \\
\hline $\begin{array}{l}\text { Crotalaria } \\
\text { retusa }\end{array}$ & $8.15^{\mathrm{c}}$ & $2.99^{e}$ & $0.25^{\mathrm{f}}$ & $3.91^{\mathrm{e}}$ & $1.05^{\mathrm{c}}$ & $14.70^{a}$ & $0.91^{\mathrm{c}}$ & $2.35^{\mathrm{c}}$ & $2.82^{\mathrm{c}}$ & $1.50^{\mathrm{f}}$ & $1.28^{\mathrm{c}}$ & $1.49^{\mathrm{d}}$ & $1.17^{\mathrm{d}}$ \\
\hline $\begin{array}{l}\text { Vigna } \\
\text { unguiculata }\end{array}$ & $10.01^{\mathrm{b}}$ & $6.16^{\mathrm{b}}$ & $12.25^{\mathrm{a}}$ & $8.92^{\mathrm{c}}$ & $0.39^{f}$ & $12.10^{\mathrm{b}}$ & $0.00^{\mathrm{f}}$ & $2.66^{\mathrm{b}}$ & $3.46^{a}$ & $2.52^{\mathrm{b}}$ & $1.59^{\mathrm{a}}$ & $2.10^{c}$ & $1.50^{\mathrm{b}}$ \\
\hline $\begin{array}{l}\text { Centrosema } \\
\text { molle }\end{array}$ & $6.20^{\mathrm{d}}$ & $3.75^{\mathrm{d}}$ & $3.81^{\mathrm{d}}$ & $13.76^{\mathrm{b}}$ & $0.61^{\mathrm{e}}$ & $13.00^{\mathrm{b}}$ & $0.90^{c}$ & $3.92^{\mathrm{a}}$ & $3.05^{\mathrm{b}}$ & $2.22^{\mathrm{c}}$ & $0.76^{\mathrm{d}}$ & $2.46^{\mathrm{b}}$ & $1.40^{\mathrm{c}}$ \\
\hline $\begin{array}{l}\text { Gliricidia } \\
\text { sepium }\end{array}$ & $7.69^{\mathrm{dc}}$ & $4.62^{\mathrm{c}}$ & $3.86^{\mathrm{d}}$ & $15.24^{a}$ & $1.92^{\mathrm{b}}$ & $7.10^{c}$ & $0.94^{\mathrm{c}}$ & $2.17^{\mathrm{d}}$ & $2.17^{\mathrm{d}}$ & $1.90^{\mathrm{d}}$ & $0.78^{\mathrm{d}}$ & $1.35^{\mathrm{e}}$ & $0.46^{\mathrm{f}}$ \\
\hline
\end{tabular}

Means with the same superscript down the column were not significantly different $(\mathrm{P}<0.0001)$ 
244

petals: standard: $3.20-4.50 \mathrm{~cm}$ long and $2.40-3.50 \mathrm{~cm}$ wide, keel: $1.80-2.50 \mathrm{~cm}$ long and $0.6-0.90 \mathrm{~cm}$ wide, wing: $1.90-2.90 \mathrm{~cm}$ long and $1.00-1.80 \mathrm{~cm}$ wide, bright lilac with violet veining, papilionaceous in shape, 5 in number, pedicel $0.70-1.20 \mathrm{~cm}$ long (Fig. 9F).

Fruit: legume, aristate at apex, $8.20-16.60 \mathrm{~cm}$ long and $0.40-0.80 \mathrm{~cm}$ wide, green when fresh, brown when dry, glabrous, one row of seed per fruit (Fig. $9 \mathrm{H}$ ).

Seed: bean-shaped, light brown to dark brown with black patches, shinny, glabrous, $6-8$ seeds per fruit (Fig. 9E).

Gliricidia sepium (Jacq.) Walp. (Fig. 10)

Habit: tree (Fig. 10A).

Lifespan: perennial.

Leaf: compound-unipinnate, leaflet; $4.30-10.60 \mathrm{~cm}$ long and $3.20-6.30 \mathrm{~cm}$ wide, elliptic, obtuse at base, acute at apex, entire at margin, green, glabrous, exstipulate, petiolate, petiole $3.10-4.60 \mathrm{~cm}$ long (Fig. 10B).

Stem: erect, woody, solid, brown with white spots, cylindrical, glabrous (Fig. 10C).

Inflorescence: terminal panicle (Fig. 10D).

Flower: zygomorphic, sub sessile, calyx tube: green and reddish brown, campanulate, gamosepalous, glabrous; petals: standard: $2.10-2.20 \mathrm{~cm}$ long and $2.10-2.20 \mathrm{~cm}$ wide, keel: $1.80-2.00 \mathrm{~cm}$ long and $0.70-0.90 \mathrm{~cm}$ wide, wing: $1.30-1.40 \mathrm{~cm}$ long and $0.40-0.50 \mathrm{~cm}$ wide, pinkish purple with white at the middle of the standard, papilionaceous, 5 in number, pedicel $0.70-1.10 \mathrm{~cm}$ long (Fig. 10E and F).

Fruit: legume, flat, slightly ribbed, elongated, obtuse at apex, base narrow than apex, $12.30-19.50 \mathrm{~cm}$ long and $1.50-2.20 \mathrm{~cm}$ wide, green when fresh, brown when dry, glabrous, one row of seed per fruit (Fig. 10G).

Seed: orbicular with prominent hilum, dark brown and coarse, not pubescent, $4-10$ seeds per fruit (Fig. 10H).

\section{Discussion}

Many authors have stressed morphological characters as taxonomic tools. These include Hutchinson and Dalziel (1958), Terrel and Winters (1974), Isawumi (1985), Adedeji and Illoh (2005) and Adedeji (2005, 2006).

El-Gazzar et al. (2013) reported that the only common feature of all classificatory systems of the Papilionoideaeto date is the recognition of tribes and sub-tribes on the basis of a limited range of floral traits, with greater emphasis on petal morphology and stamen arrangement. Such a limited number of characters were used often singly to distinguish between chunky assemblages of genera.

In the current study, additional traits, further from those reported by Hutchinson and Dalziel (1958), which are quite diagnostic for the taxonomy of the subfamily, were herein reported. Additional traits which previous researchers have not reported include life span; leaf/leaflet apex, base, margin and pubescence; stem type, colour, shape and pubescence; sepal colour and pubescence; nature of margin of petal standard and presence or absence of pedicel; fruit colour, pubescence, tip and shape; seed colour, shape, surface and presence or absence of prominent hilum on the seed.

The morphological characters used for this study are sufficient in establishing the relationships among the species of Papilionoideae studied. Thirty eight qualitative and thirteen quantitative traits employed have been reported in the current study.

Joelri et al. (2011) reported in their work on Crotalaria in South India that leaves, simple or compound, is a major feature in developing taxonomic key based on morphological characters. In this study, leaves were compound except in the case of Crotalaria retusa, which was the only species with simple leaves; this can be used to delimit it from the other species. Gliricidia sepium (Tribe Robinieae) which was the only species that had compoundonce pinnate leaves, can further be separated from the other species which had compound-trifoliate leaves. This also justifies its separation from other tribes. Rahman and Rahman (2012) used leaf shape to group Desmodium alatum and Desmodium auriculatum together and separated them among 14 species of Desmodium studied. Adedeji (2006) also reported that the shape of the upper leaves can be used in the taxonomy of the genus Emilia. Leaf shape was also used by Joelri et al. (2011) to discriminate Crotalaria verrucosa from the rest of Crotalaria species studied in South India.

In the hereby study, leaf/ leaflet shapes can also be used to delineate the species of Desmodium from one another and other species studied. Leaflets of Desmodium tortuosum were ovate, those of Desmodium scorpiurus were elliptic, while those of Desmodium adscendens were obovate in shape. Leaflets of Mucuna pruriens and Calopogonium mucunoides were wide ovate to wide elliptic. Crotalaria retusa in the tribe Crotalarieae was the only species with oblanceolate leaf shape and this also delineated it from other tribes.

The stem of Crotalaria retusa was cylindrical in shape, with vertical ridges, while that of Cajanus cajan was cylindrical, with distinct angles. Desmodium tortuosum was the only species that had ringed stem. Desmodium scorpiurus had triangular shaped stem, while that of Centrosema molle was twisted. Crotalaria retusa was the only species with hollow stem, whereas all others had solid stems. These characters of stem can be used to delimit the taxa studied.

Variation also occurred within the petal colour among the species. However, all Desmodium species studied had pinkish purple flowers. This supports their grouping into the same tribe Desmodieae. Joelri et al. (2011) separated Crotalaria laburnifolia and Crotalaria pallida from other Crotalaria species studied using reddish brown stripes at the keel petals. In this study, bright yellow petals with reddish brown veining on the standard were recorded for Cajanus cajan and Crotalaria retusa. This can be used to group them apart; even more, standard petal of Cajanus cajan was indented at margin and this can be used to separate it from Crotalaria retusa and other species which were entire at margin.

Daiane et al. (2014) reported the importance of the form of loment margin as identification key for Desmodium species studied in Santa Catarina, Brazil. Fruits were legume except in Desmodium species from the tribe Desmodieae, which were lomentum, and this delimited the tribe from other tribes studied. However, Desmodium adscendens can be separated from other Desmodium species because its 
fruit were deeply indented on one side only, while others were indented on both sides. There were variations also in the shape, colour and surface of seeds. These characters can also delimit the species. Crotalaria retusa had reniform (kidney-shaped) seeds, Desmodium scorpiurus was cylindrical, while others were bean shaped. This can be of taxonomic value.

The current study reports that Crotalaria retusa was the only species with simple leaves, oblanceolate leaf shape, cuneate leaf base, sub sessile leaves, hollow stem and reniform seed shape. These distinct characters justify the delimitation of Crotalaria retusa in the tribe Crotalarieae from the other tribes in the sub-family. Quantitatively, Desmodium adscendens had the lowest leaf size (both length and width). Petiole length of the pair Mucuna pruriens and Calopogonium mucunoides, as well as the pair Centrosema molle and Gliricidia sepium were not significantly different. These characters can be used to separate these taxa from other species. Desmodium tortuosum had the longest pedicel length.

As regards petal values, petal standard length, petal keel length and petal wing width are more useful in separating or delineating the genera and tribes than the values for leaf length and width, petiole length, fruit length and width, number of seeds per fruit and pedicel length. These petal values were not significantly different within the three species in the genus Desmodium, but were significantly different between the other genera studied. However, the leaf length, petiole length, fruit length, number of seeds per fruit and pedicel length values can be used to delineate the species within the genus Desmodium as the values were significantly different within each character for the Desmodium species.

\section{Conclusions}

This study hereby reported that the important characters that can be used in establishing taxonomic relationship in the sub-family Papilionoideae are leaf type, leaf shape, leaf base, petiole type, stem shape, petal colour, petal margin and seed shape.

\section{References}

Adedeji O (2005). Comparative morphological studies of cultivated (Lycopersicon esculentum Mill.) and wild (Lycopersicon pimpinellifolium (Jusl.) Mill.) species of tomato, sub-genus Esculentum. Science Focus 10(3):17-23.

Adedeji O, Illoh HC (2005). Vegetative and floral morphological studies of some species of Hibiscus Linn. in Nigeria. Ife Journal of Science 7(1):113.

Adedeji O (2006). Morphological studies in the genus Emilia (Senecioneae, Asteraceae). Botanica Lithuanica 12(1):19-24.

Alexander K (2004). Abaxial foliarvestiture of Desmodium Desv. (Fabaceae) in North Carolina and vegetativer of the species. Vulpia 3:140-172.

APG (2012).Angiosperm phylogeny group. Fabales. Retrieved 2014Sept 5 from www.mobot.org/mobot/research/APweb/orders/fabalesweb.htm].
Cullen J, Knees SG, Cubey HS (2011). The european garden fora, vol 3, Angiospermae - Dicotyledons: Resedaceae to Cyrillaceae, 2nd ed. Cambridge University Press.

Daiane MF, Ademir R, Roseli L, da Costa B, Marisa S (2014). Morphological and micromorphological characteristics of Desmodium fruits (Leguminosae: Papilionoideae). Revista de Biologia Tropical 62(4):1597-1608.

Dutta SC, Mukerii B (1952). Pharmacognosy of Indian leaf drugs. Govt. of India Press, Calcutta.

Duane I, Paul EB (2012). Origin of Fabales and its relationship with other plant families. Encyclopaedia Britannica Inc. Retrieved on 2012 Oct 12 from

http://www.britannica.com/EBchecked/topic/199654/Fabales/ 72896/Classification-of-Fabaceae

El-Gazzar A, El-Ghani MA, El-Husseini N, Khattab A (2013). Classification of the Leguminosae-Papilionoideae: A Numerical Re-assessment. Notulae Scientia Biologicae 5(4):499-507.

Gurcharan S (2004). Plant systematics: An integrated approach. Plant Systematics: Theory and Practice. Science Publisher Inc, New Hampshire, USA.

Hutchinson J, Dalziel JM (1954). Flora of west ttropical Africa. Vol I, Part 1, (2nd ed). Crown Agents for Oversea Government, London pp 505-587.

Hutchinson J, Dalziel JM (1958). Flora of west tropical Africa. Vol I, Part 2, (2nd ed) Whitefriars Press, London pp 343-348.

International Legume Database \& Information Service (ILDIS) (2005). Sub-family Papilionoideae. Version 10.01, Retrieved on 2014 Sept 2 from http://www.ildis.org/.

Isawumi MA (1985). The taxonomy of the genus Capsicum (Solanaceae) in West Africa. Nigeria Journal Science 17:1-7.

Joelri LMR, John SB, Prabhu S, Senthilkumar SR (2011). Identification of agronomically valuable species of Crotalaria based on phonetics. Agriculture and Biology Journal of North America 2(5):840-847.

Metcalfe CR, Chalk L (1979). Anatomy of the Dicotyledons, 2nd edn, Systematic anatomy of the leaf and stem, with a brief history of the subject. Clarendon Press, Oxford pp 63-75.

Polhill RM, Raven PH (1981). Advances in legumes systematics. Royal Botanic Gardens, Kew England.

Rahman MO, Rahman MDZ (2012). Morphometric analysis of Desmodium Desv. in Bangladesh. Bangladesh Journal of Botany 41(2):143-148.

Sayantan T, Amal KM (2004). Taxonomic diversity in epidermal cells (stomata) of some selected Anthophyta under the order Leguminales (Caesalpniaceae, Mimosaceae and Fabaceae) based on numerical analysis: A systematic approach. IJSN 3(4):788-798.

Terrel EE, Winters HF (1974). Changes in scientific names for certain crops plants. Horticulture Science 9(4):324-325.

Watson L, Dallwitz MJ (1999). The families of flowering plants: descriptions, illustrations, identification, and information retrieval. University of New Orleans. 